discussed well in terms of accelerated observers in special relativity. But to master Lilley's "near equations" seems to require as much effort as would elementary calculus proper. Some limitations of the approach are apparent from the author's speculations on an alternative theory. This is incompatible with the precession of Mercury, but the reader (or author) is not provided with the confidence to do the relevant short calculation. Nor does one get any feeling for the results of the theory in cosmology, black holes or gravitational waves.

There are some mistakes: for example, a too-simple proof that bodies cannot move with the speed of light, and a peculiar notion of covariant tensors, which arises from confusing two meanings of "covariant". And amongst the more serious errors, two incompatible equations for the same gravitational force arise from an inconsistent approximation.

But for me the achievement outweighs these flaws of execution. Of the greatest importance is the demonstration, through a large number of well-made teaching points, that mathematics, the language of science, is available to anyone with sufficient motivation and the right guide.

D.J. Raine is a Lecturer in Theoretical Astrophysics at the University of Leicester. His books include Einstein and Relativity (Priory Press, 1975) and The Isotropic Universe (Adam Hilger, 1981).

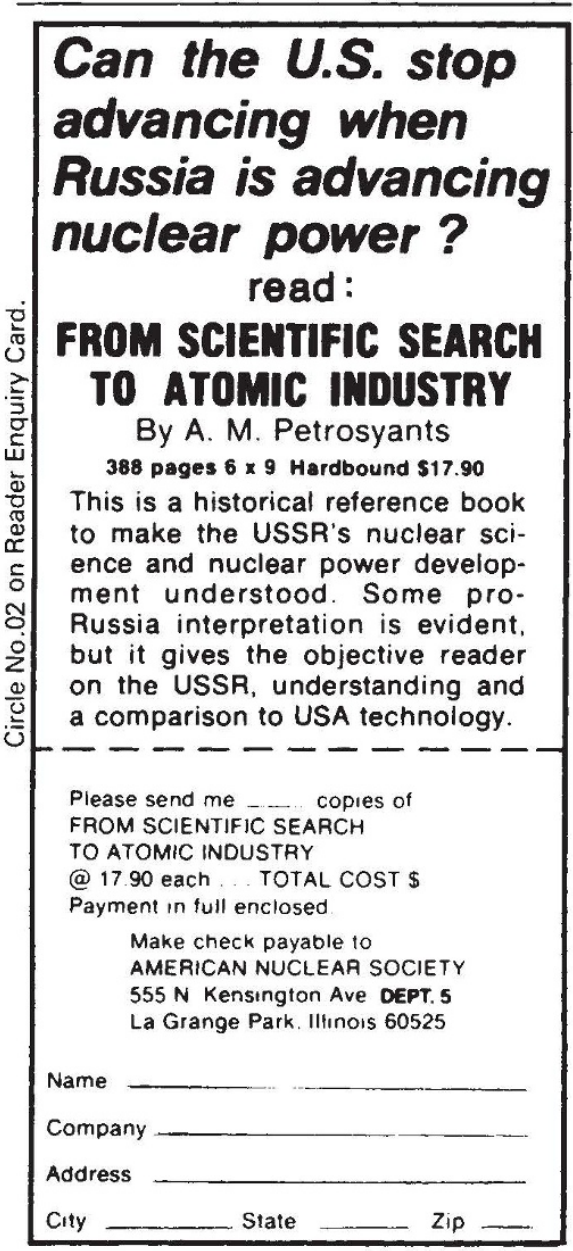

\section{From the shadows, everyman's Maxwell}

R.V. Jones

James Clerk Maxwell: A Biography. By

Ivan Tolstoy. Pp.194. ISBN 0-86241-010-X. (Canongate, Edinburgh: 1981.) £9.95.

FOR many years there hung in one of the corridors of the Electrical Laboratory at Oxford a photographic portrait of someone whom none of the staff could identify. Presumably it had been placed there by the Professor of Physics, J.S.E. Townsend; but after his death, the identity of the portrait was forgotten. It was, in fact, of James Clerk Maxwell.

Maxwell has been almost as little known in his native Scotland, where his memory has been heavily overshadowed by that of Kelvin - and this despite the fact that Einstein himself credited Maxwell with having brought about the greatest revolution in physics since the time of Newton. Not only did Maxwell create the electromagnetic theory which led to the discovery of radio waves and of electromagnetic radiation pressure, but he also formulated his distribution law governing the molecular velocities in gases, the basis of automatic control theory and the principles of kinematic design; and he demonstrated the first colour photograph.

The current year has seen the one hundred and fiftieth anniversary of his birth on 13 June 1831, while less than two years ago there occurred the centenary of his death. The commemoration of these anniversaries has coincided with increasingly wide-spread recognition of his tremendous contributions, and with papers and books describing aspects of his life and work, notably C.W.F. Everitt's short but carefully written biography (Charles Scribner's Sons, 1975) and the exhibition of Maxwell memorabilia at last year's Royal Society conversazione.

Now we are presented with another short and very readable biography, by Ivan Tolstoy, who in his preface sets out his objective:

For physicists the name of James Clerk Maxwell ranks next to Newton and Einstein. Yet among non-scientific people Maxwell's image is surprisingly faint. It is hoped the present book will help remedy that injustice. This pretends neither to be a definitive biography, nor a work of historical scholarship. It is, rather, a book for the lay reader.

Even so, and although the author has, as he says, drawn largely on secondary sources, more specialist readers will find that he has thrown shafts of light on Maxwell's character and methods of working. And in well-drawn quotations from Maxwell's own writings he has followed one of his subject's own precepts:

It is a great advantage to the student of any subject to read the original memoirs of that subject, for science is always most completely assimilated in the nascent state.
One of the rare points in the book which could be questioned concerns the process famous displacement current in a vacuum. Tolstoy says that "There could be no physical or logical justification for keeping the concept in the context of a vacuum". This might be true if Maxwell regarded a vacuum as containing absolutely nothing, but in his 1864 paper he stated his belief in "an aethereal medium filling space"' and in its having a "small but real density"; and he evidently retained this view throughout his work, for towards the end of the Treatise of 1873 he repeated "We must therefore regard the medium as having a finite density" (para.782). So Maxwell's train of thought was both physical and logical. by which Maxwell came to conceive the

James Clerk Maxwell, the portrait that hung in the Electrical Laboratory at Oxford.

Tolstoy remarks elsewhere that "Both Einstein and Maxwell were stronger in physical intuition than in mathematics", which struck an immediate resonance in my memory with something Einstein said in conversation with F.A. Lindemann during his visit to Oxford in 1931 . He was commenting on the popular description of himself as a mathematician: "I am not a mathematician. I am a physicist - if I had been a mathematician I could not have done what I have". But, of course, both Einstein and Maxwell had an impressive command of mathematics when they needed it, and Maxwell was outstandingly strong in geometrical reasoning.

Tolstoy's short account is both penetrating and sympathetic regarding Maxwell's personal relationships, of which it gives a warmly human picture; and it contains many thoughtful comments on Maxwell's physics and the context in which his advances were made. It is a book well worth reading.

$R . V$. Jones is Professor in the Department of Natural Philosophy at the University of Aberdeen, and author of The Complete Physicist: James Clerk Maxwell 1831-1879 (Yearbook of the Royal Society of Edinburgh, $5-23 ; 1980)$. 